\title{
I MISTERI CHE CIRCONDANO LA MALATTIA DI KAWASAKI: UNA RECENSIONE DELLA LETTERATURA
}

\section{ARTICOLO ORIGINALE}

ROSSI, Karoline ${ }^{1}$, MOREIRA, Danilo José Silva ${ }^{2}$, FONSECA, Juliana Brito da ${ }^{3}$, VASCONCELOS, Suzana dos Santos ${ }^{4}$, OLIVEIRA, Vinicius Faustino Lima de ${ }^{5}$, DIAS, Claudio Alberto Gellis de Mattos $^{6}$, OLIVEIRA, Euzébio de ${ }^{7}$, DENDASCK, Carla Viana $^{8}$, ARAÚJO, Maria Helena Mendonça de ${ }^{9}$, FECURY, Amanda Alves ${ }^{10}$

ROSSI, Karoline. Et. al. I misteri che circondano la malattia di Kawasaki: una recensione della letteratura. Revista Científica Multidisciplinar Núcleo do Conhecimento. Anno 06, Ed. 04, Vol. 05, pp. 52-64. Aprile. ISSN: 2448-0959, Link di accesso: https://www.nucleodoconhecimento.com.br/salute/malattia-di-kawasaki, DOI: 10.32749/nucleodoconhecimento.com.br/salute/malattia-di-kawasaki

\section{RIEPILOGO}

La malattia di Kawasaki (DK) o sindrome del linfonodo mucocutaneo è una vasculite sistemica, che colpisce principalmente i bambini sotto i cinque anni di età con discendenza asiatica, ma può anche raggiungere altre fasce d'età, così come qualsiasi altra razza. II quadro clinico di DK ha tre fasi: stadio febbrile acuto, in cui la

\footnotetext{
${ }^{1}$ Accademico medico. Università Federale di Amapá (UNIFAP).

${ }^{2}$ Studioso di medicina. Università Federale di Amapá (UNIFAP).

${ }^{3}$ Accademico medico. Università Federale di Amapá (UNIFAP).

${ }^{4}$ Accademico medico. Università Federale di Amapá (UNIFAP).

${ }^{5}$ Studioso di medicina. Università Federale di Amapá (UNIFAP).

${ }^{6}$ Biologo, Dottore di Ricerca in Teoria e Comportamento, Professore e ricercatore del Corso di Laurea in Chimica dell'Istituto di Educazione Di Base, Tecnica e Tecnologica di Amapá (IFAP).

${ }^{7}$ Biologo, PhD in Malattie Tropicali, Professore e ricercatore del Corso di Educazione Fisica, Università Federale di Pará (UFPA).

${ }^{8}$ Teologo, Dottore di Ricerca in Psicoanalisi, ricercatore presso il Centro di Ricerca e Studi Avanzati (CEPA).

${ }^{9}$ Dottore, Master in Scienze dell'Insegnamento e della Salute, Professore e ricercatore del Corso di Medicina del Campus Macapá, Università Federale di Amapá (UNIFAP).

${ }^{10}$ Biomedicale, PhD in Malattie Tropicali, Professore e ricercatore del Corso di Medicina del Campus Macapá, Università Federale di Amapá (UNIFAP).
}

RC: 82507

Disponível em: https://www.nucleodoconhecimento.com.br/salute/malattia-dikawasaki 
congestione congiuntivale, la mucosite orale, l'eritema, la sfaldamento, l'eruzione polimorfica e la linfoadenopatia laterale, compaiono come sintomi principali; lo stadio subacuto, che si verifica alla fine della febbre, e porta alla comparsa di pelle che sfalda negli arti, artrite, artralgia e trombocitosi e infine lo stadio di convalescenza che si verifica quando i sintomi si dissipano quasi e continuano fino alla loro normalizzazione. II trattamento più utilizzato si verifica dalla somministrazione di immunoglobulina endovenosa, che per una migliore prognosi della patologia dovrebbe essere iniziata precocemente.

Parole chiave: Sindrome del linfonodo mucocutaneo, Vasculite, Arterite, Febbre, Kawasaki.

\section{INTRODUZIONE}

La malattia di Kawasaki (DK) è una vasculite sistemica che colpisce i vasi di piccolo e medio calibro e colpisce principalmente i bambini sotto i 5 anni di età, essendo una delle ragioni delle malattie cardiache durante l'infanzia (HUANG et al., 2015; MAGALHÃES, 2008). L'eziologia di DK, cioè gli agenti causali di questa malattia, non sono noti. Tuttavia, alcuni aspetti clinici ed epidemiologici propongono possibili ragioni infettive (FERRONATO et al., 2010).

DK è la seconda vasculite più comune in età pediatrica, che colpisce principalmente $\mathrm{i}$ bambini di età inferiore a mezzo decennio (90\%). È raro nei bambini sotto i 6 mesi e più grandi di 8 anni, che, tuttavia, sono più inclini allo sviluppo di aneurismi coronari. Su scala globale, c'è una variazione nell'occorrenza, con il Giappone che è il paese più colpito, con un'incidenza annuale da 110 a 150 casi ogni 100.000 bambini sotto $i$ 5 anni. Considerando il calcolo dei tassi di incidenza in ogni 100.000 bambini sotto $i$ 5 anni di età, negli Stati Uniti d'America (USA) il DK è la causa più comune di malattie cardiache acquisite dall'infanzia, che vanno da 9,1 a 32,5 casi, che si verificano più frequentemente in quelli dei discendenti delle isole asiatiche e del Pacifico $(32,5)$, intermedi negli afroamericani $(16,9)$ e ispanici $(11,1)$ e non comuni

RC: 82507

Disponível em: https://www.nucleodoconhecimento.com.br/salute/malattia-dikawasaki 
nei caucasici (9,1) (ALMEIDA et al. , 2010; CASTRO; URBANO; COSTA, 2009; RODRIGUES et al, 2017).

DK ha una prevalenza più elevata nella popolazione asiatica, con particolare attenzione al giapponese, che colpisce principalmente i bambini. C'è un rapporto di un'incidenza annuale in Giappone e Corea fino a 100 casi ogni 100.000 abitanti, mentre nella razza caucasica l'occorrenza è compresa tra 6 e 10 casi ogni 100.000 abitanti sotto l'età di mezzo decennio. In America Latina, si stima che ci siano 3 casi ogni 100.000 abitanti, dando così prova di una minore frequenza di questa patologia nelle popolazioni non asiatiche che non discendono dagli asiatici (SCARDINA et al., 2007). Per quanto riguarda il tasso di mortalità associato alla DK, è molto basso, in quanto è inferiore allo 0,1\% (BARCA et al., 2019).

L'incidenza del DK varia geograficamente nel mondo, essendo più diffusa nei discendenti giapponesi, suggerendo una predisposizione genetica della malattia, oltre a possibili agenti che possono essere collegati alla posizione geografica, come i batteri (CASTRO; URBAN; COSTA, 2009; RODRIGUES et al, 2017).

La variazione stagionale nell'incidenza della DK è ben riconosciuta, ma i tassi cambiano da un paese all'altro. Nel Regno Unito, in Australia e negli Stati Uniti, vi è un numero maggiore di casi in inverno e in primavera. In Cina e Corea, in primavera e in estate. Negli Stati Uniti e nel Regno Unito, l'aumento dei tassi è più correlato alla variazione dei periodi di pioggia che alla variazione della temperatura. In Brasile, uno studio condotto con 70 pazienti con DK ha mostrato un record più elevato della malattia in coincidenza con periodi di maggiore incidenza di malattie infettive da maggio a giugno (inizio della siccità) e novembre e dicembre (piogge precoci) (MAGALHÃES; ALVES, 2017).

Una persona con DK sviluppa caratteristiche sintomatologiche che portano alla presentazione di un quadro clinico, che consiste nellinsieme di segni e sintomi manifestati dal paziente (KAYIRAN; DINDAR; GURAKAN, 2010).Pertanto, il quadro

RC: 82507

Disponível em: https://www.nucleodoconhecimento.com.br/salute/malattia-dikawasaki 
clinico di DK è caratterizzato da linfoadenopatia cervicale, febbre, tonsillite, gonfiore degli arti, eruzione cutanea, prurito e sfaldamento (ATIK, 2007; SCARDINA et al., 2007). Inoltre, DK può causare infiammazione generalizzata, colpendo alcuni organi causando cardiomiopatia infiammatoria, meningite sterile, epatite, adenolinfite, pericardite e angite (CASTRO; URBAN; COAST, 2009).

Castro, Urbano e Costa (2009) indicano una relazione tra la vasculite causata da DK e il coinvolgimento di alcuni organi, che consistono nel raggruppamento di tessuti responsabili del mantenimento dell'organismo.

DK è stato segnalato per la prima volta nel 1967 dal medico giapponese Tomisaku Kawasaki, che lo ha definito come "sindrome del linfonodo mucocutaneo". Durante questo periodo sono stati segnalati 50 casi negli anni dal 1961 al 1967. In Giappone, l'occorrenza è più elevata e la distribuzione globale ha la sua prevalenza variabile (ALMEIDA et al., 2010; SCARDINA et al., 2007; MAGALHÃES; ALVES, 2017).

Nello scenario attuale, sono già stati stabiliti alcuni dati epidemiologici sulla DK, consentendo un'analisi più accurata dei fattori che intervengono nella diffusione e diffusione delle malattie. Molti studi sono stati condotti al fine di comprendere la fisiopatologia della malattia, che consiste in una serie di cambiamenti anomali causati nel corpo (CASTRO; URBANO; COSTA, 2009).

In uno studio, sono state trovate prove di DK e l'avanzamento di altre comorbidità, che si riferisce al fatto che due o più malattie sono allo stesso tempo in un individuo, quindi le complicazioni di DK sarebbero l'evoluzione sfavorevole di questa associazione (SCARDINA et al., 2007).

Per migliorare la cura e la qualità della vita del paziente con DK, sono stati sviluppati trattamenti, che consistono in modi di prendersi cura di un paziente (CASTRO; URBANO; COSTA, 2009).

RC: 82507

Disponível em: https://www.nucleodoconhecimento.com.br/salute/malattia-dikawasaki 


\section{OBIETTIVI}

Dettagliare i possibili agenti causali, la fisiopatologia, i principali organi e strutture anatomiche colpite dalla malattia di Kawasaki (DK).

Per verificare le principali caratteristiche del quadro clinico di un individuo per la diagnosi, le principali complicanze associate e i trattamenti disponibili per i pazienti con DK.

\section{METODO}

Si tratta di una ricerca bibliografica condotta nei database, Scielo, Biblioteca Digitale Brasiliana di Tesi e Tesi, LILLACS e PubMed, utilizzando parole chiave o le loro associazioni: malattia di Kawasaki-Kawasaki.

I parametri di inclusione utilizzati nella preparazione delle ricerche sono stati la disponibilità completa online, l'approccio diretto sulla malattia di Kawasaki (DK) o su alcuni aspetti pertinenti a questa malattia. Nella banca dati Scielo c'erano filtri di testo delle produzioni scritte in inglese e portoghese, nelle altre banche dati questo criterio non è stato utilizzato. I parametri di esclusione applicati sono stati duplicati ed eseguiti prima del 2000.

Un'analisi è stata fatta nei database menzionati per elencare gli articoli relativi a DK in letteratura. Da una precedente lettura dei titoli e degli abstract delle produzioni scientifiche trovate, gli studi che non corrispondevano a ciò che ci si aspettava costituisse questa recensione sono stati scartati. Sono stati letti i testi completi che hanno attraversato le fasi precedenti, selezionando per questa recensione quelli che hanno affrontato gli aspetti rilevanti di DK.

\section{RISULTATI}

\section{POSSIBILI AGENTI CAUSALI DI DK}

RC: 82507

Disponível em: https://www.nucleodoconhecimento.com.br/salute/malattia-dikawasaki 
La causa della malattia di Kawasaki è ancora sconosciuta. Tuttavia, le sue caratteristiche cliniche ed epidemiologiche alimentano l'ipotesi che un agente infettivo sia la causa scatenante, che produce cambiamenti nelle cellule, nei tessuti e negli organi di individui con predisposizione genetica. Questa ipotesi è la più probabile, ma non ha ancora prove (CASTRO; URBANO; COSTA, 2009).

Una delle teorie è quella del coronavirus NL-63, rilevata per mezzo di una reazione a catena della polimerasi (PCR) nei fluidi delle vie aeree di 11 pazienti con DK e solo in 1 dei 22 pazienti di controllo. Tuttavia, altri 5 studi successivi non hanno trovato coronavirus in campioni di tessuto respiratorio o nasofarito da pazienti, rendendo impossibile per questo virus essere la causa scatenante (CASTRO; URBANO; COSTA, 2009).

Un'altra teoria è quella della stimolazione immunologica da parte di superantigeni batterici come le tossine stafilococciche e streptococciche. Una terza teoria è immunologica, che propone che il DK sia causato da una risposta immunitaria aberrante in individui geneticamente predisposti (MAGALHÃES; ALVES, 2017).

\section{FISIOPATOLOGIA DI DK}

Le alterazioni riscontrate in DK, specialmente quelle istologiche, caratterizzano un quadro di angiite sistemica generalizzata, che colpisce principalmente vasi di calibro mediano, in particolare le arterie coronarie. In diversi organi si possono osservare cambiamenti infami, come nel miocardio, nel pericardio, nei vasi sanguigni, nelle meningi, nel polmone, nei linfonodi e nel fegato (CASTRO; URBANO; COSTA, 2009).

\section{PRINCIPALI ORGANI ANATOMICI E STRUTTURE COLPITE DA DK}

DK colpisce organi e arterie, principalmente vasi di medio calibro da vari sistemi del corpo umano, come cardiovascolare, polmonare, gastrointestinale (intestino e

RC: 82507

Disponível em: https://www.nucleodoconhecimento.com.br/salute/malattia-dikawasaki 
cistifellea sono i principali organi colpiti) e il sistema nervoso centrale (SNC). Tuttavia, va sottolineata l'implicazione che questa malattia ha nel cuore, con la formazione di aneurismi coronari (CASTRO; URBANO; COSTA, 2009).

Altre arterie possono anche essere colpite, come: aneurisma aortico, con un maggior numero di casi segnalati che compromettono l'aorta addominale; aneurisma dell'arteria ascellare; aneurisma dell'arteria brachiocefala; aneurismi delle arterie iliache, femorali; aneurisma dell'arteria renale e ostruzione dell'arteria oculare (ALVES et al., 2011).

\section{CARATTERISTICHE PRINCIPALI DEL QUADRO CLINICO DI UN INDIVIDUO PER LA DIAGNOSI DI DK}

La febbre, che segna la fase acuta della malattia, è solitamente elevata (superiore a $39^{\circ} \mathrm{C}$ ) e ai picchi, diminuendo temporaneamente di intensità della durata di circa sette-quattordici giorni, e può raggiungere tre e/o quattro settimane se non esiste un trattamento appropriato. II giorno in cui inizia la febbre è considerato il primo giorno della malattia. Tuttavia, alcuni pazienti hanno altre manifestazioni classiche prima della febbre, come: congiuntivite non purulente; linfonodo cervicalemegaly; etitemi e/o edemi sulle mani e sui piedi; exanthetem escalatiniforme, morbiliforme o polimorfico; lingua framboesiforme, eritema ed edema orofaringe, fessure ed eritema labiale (CASTRO; URBANO; COSTA, 2009).

Cambiamenti nelle estremità (mani e piedi) con eritemi e / o edema compaiono anche nella fase acuta, ed l'edema è molto doloroso ed è associato all'eritema palmoplantare con vasculite a portata di mano che dopo un periodo di una o due settimane, già in fase subacuta, inizia a sbucciare, a partire dalla regione periungueale nel dito del guanto (MAGALHÃES; ALVES, 2017).

La congiuntivite bilaterale non essudativa colpisce in particolare la congiuntiva bulbare rispetto alla congiuntiva palpebrale e tarsale ed è indolore. A volte è

RC: 82507

Disponível em: https://www.nucleodoconhecimento.com.br/salute/malattia-dikawasaki 
accompagnato da iridociclite, un'infiammazione della parte anteriore dell'occhio, con risoluzione rapida e poco associata alla sensibilità alla luce (CASTRO; URBANO; COSTA, 2009).

L'eruzione polimorfica è un insieme di eruzioni cutanee che si verificano nei primi giorni della malattia, colpendo principalmente il tronco e le estremità, senza vescicole apparenti o vescicole. Hanno una presentazione variabile (eruzione urticariforme, morbiliforme, maculopapulare) o diffusa, simile alla scarlattina (ALMEIDA et al., 2010).

Cambiamenti nelle labbra e nella cavità orale 9 casi su 10 nella fase acuta con intensa iperemia labiale e orofaringe, fessure, mucosa secca e sanguinamento delle labbra. Le papille della lingua possono diventare prominenti e a volte possono essere osservate ulcerazioni orali con orofaringe essudato. Per quanto riguarda la linfoadenomegali cervicale, un altro criterio diagnostico, il paziente deve presentare almeno un linfonodo con un diametro maggiore o uguale a $1,5 \mathrm{~cm}$, comunemente unilaterale, doloroso e sodo, nel triangolo cervicale anteriore (ALMEIDA et al., 2010; MAGALHÃES; ALVES, 2017).

\section{LE PRINCIPALI COMPLICAZIONI ASSOCIATE A DK}

Nei pazienti con DK, tre tipi di vasculopatie sono identificati in diversi momenti dell'evoluzione della malattia: arterite necrotizzante, arterite subacuta / cronica e vasculite di proliferazione miofibroblastica luminare. Nella fase acuta della malattia, spicca l'arterite necrotizzante, che ha il suo completo processo patologico nelle prime due settimane dopo l'inizio della condizione febbrile, specialmente nelle arterie coronarie, che possono determinare la formazione di aneurismi giganti suscettibili allo sviluppo di trombosi e rotture. Nella fase subacuta, viene verificato che il processo di vasculite subacuta inizia 2 settimane dopo l'inizio della febbre e può colpire tutti i vasi sanguigni, in particolare il segmento centrale delle arterie muscolari medie, come le arterie coronarie. Nella fase cronica della malattia, si verifica una

RC: 82507

Disponível em: https://www.nucleodoconhecimento.com.br/salute/malattia-dikawasaki 
mioproliferazione fibroblastica luminare, associata alla vasculite subacuta cronica (MAGALHÃES; ALVES, 2017).

La complicanza più grave della malattia è la vasculite coronarica, con cambiamenti nelle arterie coronarie, che colpisce il $15-20 \%$ dei pazienti che non ricevono il trattamento. Queste alterazioni includono aneurismi, ectasie e stenosi delle arterie coronarie, responsabili della mortalità del $2 \%$ (ALMEIDA et al., 2010).

Nel sistema gastrointestinale, c'è vomito, diarrea, dolore addominale e ileo paralitico. All'inizio della febbre, le manifestazioni più frequenti sono vomito e diarrea e le meno comuni sono ittero, epatomegalità dolorosa, pancreatite e vescicole idropice. Nell'apparato respiratorio, le manifestazioni più frequenti sono tosse, polmonite e cambiamenti radiologici. Raramente si verifica rinorreia (MAGALHÃES; ALVES, 2017).

Nel SNC, la malattia può manifestarsi con estrema irritabilità nella fase acuta, oltre a meningite asettica, atassia, paralisi facciale e perdita dell'udito neurosensoriale. Degna di nota è anche la presenza di ganglionite e neurite nei nervi cranici e periferici, endarterite, periarterite, coriomeningite e leptomeningite, oltre all'atrofia, degenerazione con perdita di neuroni, gliosi marginale e subpendimica e formazione di noduli gliali intorno alle degenerazioni neuronali (MAGALHÃES; ALVES, 2017).

Cambiamenti comportamentali come deficit di attenzione, carenza di apprendimento, cambiamenti emotivi (labilità emotiva, paura e terrore notturno) e problemi di internalizzazione (comportamento ansioso, depressivo e aggressivo) sono stati descritti in letteratura (ALVES et al., 2011).

\section{TRATTAMENTI DISPONIBILI PER LE PERSONE AFFAFFATO DA DK}

La terapia per DK ha due scopi diversi a seconda dello stadio in cui si trova. Nella fase acuta, mira a ridurre la risposta infiammatoria nella parete dell'arteria coronarica

RC: 82507

Disponível em: https://www.nucleodoconhecimento.com.br/salute/malattia-dikawasaki 
e prevenire la vasculite e le sue conseguenze (trombosi e aneurismi). Nelle fasi subacuta e cronica, l'obiettivo è prevenire l'ischemia miocardica e l'infarto (CASTRO; URBANO; COSTA, 2009).

L'immunoglobulina endovenosa (IGEV) è il principale medicinale utilizzato in DK e il suo meccanismo d'azione rimane sconosciuto. Viene utilizzato nella fase acuta, preferibilmente nei primi 7-10 giorni dall'inizio della condizione (MAGALHÃES; ALVES, 2017).

II trattamento consiste nella somministrazione di immunoglobulina in una singola dose di $2 \mathrm{~g} / \mathrm{kg}$ in un periodo variabile di $10-12$ ore insieme alla somministrazione di acido acetilsalicilico (AAS) da 80 a $100 \mathrm{mg} / \mathrm{kg} / \mathrm{giorno}$, per via orale, 6/6 ore. L'ASA ad alte dosi ha un effetto antinfiammatorio e, a basse dosi, agisce come inibitore dell'aggregazione piastrinica, in modo che il tempo di utilizzo dell'AAS in dosi elevate rimanga fino a quando il bambino non è afebrile. Quindi, la dose viene ridotta a 3-5 $\mathrm{mg} / \mathrm{kg} / \mathrm{giorno}$ e mantenuta a questa dose mentre il paziente presenta trombocitosi e/o cambiamenti coronarici (MAGALHÃES; ALVES, 2017; RODRIGUES et al., 2017).

Tra 1 e 2 su 10 dei pazienti con DK non rispondono alla prima dose di IGEV, o con la permanenza della condizione febbrile o con la ricomparsa di esso un giorno e mezzo dopo la prima infusione. Questi pazienti sono considerati refrattari al trattamento e sono quelli con il più alto rischio di cambiamenti coronarica. In questi casi, si raccomanda la somministrazione della seconda dose di IVIG di $2 \mathrm{~g} / \mathrm{kg}$ in una singola dose. Se la febbre persiste per 36 ore, si raccomanda di utilizzare la terapia a impulsi con metilprednisolone ad una dose di $30 \mathrm{mg} / \mathrm{kg} / \mathrm{giorno}$ (dose massima $1 \mathrm{~g} / \mathrm{giorno}$ ), infusa a 1 ora, 1 ora/giorno, per 3 giorni (MAGALHÃES; ALVES, 2017).

Nel trattamento del DK nelle fasi subacuta e cronica, l'aspirina a bassa dose (3-5 mg / kg / giorno) viene utilizzata nei bambini con aneurismi piccoli e medi. In queste fasi, il trattamento mira a prevenire la trombosi (attivazione piastrinica) e la stesi del vaso. Vengono utilizzati anche altri agenti antipiastrinica (clopidogrel, ticlopidina,

RC: 82507

Disponível em: https://www.nucleodoconhecimento.com.br/salute/malattia-dikawasaki 
dipiridamolo) e, associati all'aspirina, si presentano come più concreti nel bloccare l'aggregazione piastrinica (CASTRO; URBANO; COSTA, 2009).

\section{DISCUSSIONE}

\section{CORRELAZIONE TRA POSSIBILI CAUSARS E LO SVILUPPO DI DK}

Ad oggi, l'origine di DK non è stata identificata, ma ci sono alcuni agenti, che si ritiene siano correlati allo sviluppo della patologia. Questi sono Staphylococcus aureus, estreptococos, vírus influenza, morbilivírus, paramixovírus, bunyavírus. Anche l'adenovirus e un nuovo coronavirus umano chiamato coronavirus di New Haven (Nco-NH) possono essere associati a $\mathrm{DK}$, in quanto sono già stati identificati nelle secrezioni delle vie respiratorie dei pazienti con la malattia. Tali virus e batteri potrebbero agire sullo sviluppo di DK, in modo che portino a dati causati dalla malattia, come gli aneurismi aortici (BARCA et al., 2019; CASTRO; URBANO; COSTA, 2009).

C'è un'ipotesi che associa l'esistenza di superantigeni batterici allo sviluppo del KD. Gli studi che analizzano questo suggerimento presuppongono che il superantigeno possa legarsi alla cellula che presenta l'antigene, attraverso il complesso di istocompatibilità maggiore di classe II (Classe II MHC) e il recettore dei linfociti T in siti di legame diversi da quelli degli antigeni comuni. Ciò promuoverebbe una risposta immunologica con la formazione e il rilascio di citochine pro-infiammatorie in concentrazioni eccessive, contribuendo all'evoluzione di un processo infiammatorio caratteristico di DK (CASTRO; URBANO; COSTA, 2009).

Alcune indicazioni portano a credere che una risposta $\lg A$ oligoclonale si verifichi in DK acuto. Uno studio condotto da Castro, Urbano e Costa (2009) ha eseguito l'analisi della catena alfa pesante di immunoglobuline raccolte da una grande quantità di leucociti accumulati in un sito di risposta infiammatoria della parete arteriosa dei pazienti con DK, che ha mostrato che nel campione sono stati rilevati

RC: 82507

Disponível em: https://www.nucleodoconhecimento.com.br/salute/malattia-dikawasaki 
anticorpi specifici di immunoglobulina A $(\lg A)$. Ciò dimostra l'esistenza di una risposta immunitaria correlata a DK (CASTRO; URBANO; COSTA, 2009).

L'ipotesi che DK abbia predisposizione genetica è rafforzata dall'alta incidenza tra gli asiatici e le loro rummie. Uno studio ha mostrato 67 geni legati allo sviluppo della patologia, che hanno comportato il coinvolgimento nell'endotelio, nel metabolismo lipidico, nell'attivazione di meccanismi immunologici e nel reclutamento piastrinico, supportando l'idea che esiste una relazione genetica per lo sviluppo di DK (CASTRO; URBANO; COSTA, 2009; SOTELO; GONZÁLEZ, 2007).

\section{FISIOPATOLOGIA DI DK}

La vasculite inizia con il verificarsi di gonfiore e infiammazione, che può verificarsi per un periodo variabile. I neutrofili sono presenti inizialmente, e subito dopo c'è una predominanza di linfociti $T$ citotossici CD8+ e immunoglobulina IgA (CASTRO; URBAN; COAST, 2009).

Nelle arterie coronarie ci sono cambiamenti infiammatori con la presenza di edema e necrosi delle cellule muscolari. Con questa perdita di integrità strutturale c'è la formazione di aneurismi. Inoltre, i fibroblasti proliferano e poi rimodellano, portando alla possibile presenza di sutesi, calcificazioni e trombosi (CASTRO; URBANO; COSTA, 2009; SOTELO; GONZÁLEZ, 2007).

È importante notare che il fattore infiammatorio 1 presente negli allosinnesti è fortemente aumentato nei tessuti arteriosi shimnotici di DK. Questo fattore infiammatorio 1 dell'allografto lega la risposta dell'interferone di tipo I fino all'attivazione di specifici macrofagi e linfociti $\mathrm{T}$, che assume la probabile importanza dell'interferenza di queste cellule nell'arterite da parte di DK (ROWLEY et al., 2017).

\section{COME I PRINCIPALI ORGANI E STRUTTURE ANATOMICHE SONO INFLUENZATI DA DK}

RC: 82507

Disponível em: https://www.nucleodoconhecimento.com.br/salute/malattia-dikawasaki 
DK colpisce organi e arterie, principalmente vasi di medie dimensioni, come quelli del cuore, in particolare le arterie coronarie, con la formazione di aneurismi. La malattia può anche influenzare il sistema respiratorio (polmone), attraverso la vasculite, il sistema gastrointestinale, principalmente l'intestino e la cistifellea, e il sistema nervoso (CASTRO; URBANO; COSTA, 2009).

II sistema linfatico può essere affetto da DK, poiché uno dei sintomi di DK è il gonfiore dei linfonodi cervicali. II sistema cutaneo è danneggiato da eritema cutaneo, seguito da edemi. Negli occhi può verificarsi iperemia congiuntiva non suputiva e le mucose della cavità orale possono essere colpite da mucosite orale e lesioni sulle labbra (SOTELO; GONZÁLEZ, 2007).

\section{LE PRINCIPALI CARATTERISTICHE DEL QUADRO CLINICO DI UN INDIVIDUO CON DK E COME LO INFLUENZANO}

II decorso clinico del DK può essere suddiviso in tre fasi cliniche: acuta, subacuta e convalescenza. Lo stadio febbrile acuto di solito dura da 7 a 14 giorni, caratterizzato da congestione congiuntivale, mucosite orale, eritema, eruzione cutanea flaking e polimorfica e linfoadenopatia. Lo stadio subacuto è il periodo tra la fine della febbre e il $25^{\circ}$ giorno della malattia. In questa fase, i pazienti presentano la sfaldatura della pelle negli arti, l'artrite, l'artralgia e la trombocitosi. La fase di convalescenza inizia quando i sintomi clinici iniziano a scomparire e continua fino alla normale istituzione dell'VHS (tasso di pressione sanguigna rossa), che di solito si osserva da 6 a 8 settimane dopo l'insorgenza dei sintomi. Tali fasi influenzano la vita del paziente, poiché gli impediscono di svolgere le sue attività quotidiane, che è principalmente dovuta a lesioni cutanee e sintomatologia (ALMEIDA, 2017; SCARDINA et al., 2007).

La condizione suggestiva della patologia è caratterizzata da febbre, aumento dei linfonodi cervicali e tonsillite, seguita da eritema cutaneo generalizzato, prurito ed edema degli arti e dopo alcuni giorni di sfaldamento. I test di laboratorio mostrano una fase infiammatoria attiva con alto tasso di sedimentazione, proteina C-reattiva,

RC: 82507

Disponível em: https://www.nucleodoconhecimento.com.br/salute/malattia-dikawasaki 
leucocitosi e aumento del numero di piastrine, che porta alla letargia dell'individuo interessato, poiché l'infiammazione può causare forti dolori (ALMEIDA, 2018; ATIK; FORONDA; BUSTAMANTE, 2003; FERRONATO et al., 2010; ROSSI et al., 2015).

Può anche insorgere iperemia congiuntiva non suputiva; eruzione macularepapulare; lesioni sulle labbra e cavità orale; cambiamenti nella pelle delle estremità, oltre al mormorio precordiale, difficoltà respiratorie, dolore addominale, disuria, epatomegaly, artralgia, irrigidimento del collo e convulsioni. Questi sintomi impediscono al paziente di vedere e spostarsi normalmente, poiché gli occhi sono danneggiati e il dolore causa difficoltà, per eseguire movimenti (CASTRO; URBANO; COSTA, 2009; SOTELO; GONZÁLEZ, 2007).

DK colpisce diversi pazienti con vasculite in vari organi, come è stato detto in precedenza, il polmone, l'intestino, la cistifellea, il sistema nervoso centrale, sono esempi, il che porta alla comparsa di sintomi come affaticamento, perdita di peso e dolore alle articolazioni e ai muscoli, ma il danno cardiaco è il più significativo, con la formazione di aneurismi coronari, che portano all'angina (CASTRO; URBAN; COSTA, 2009; PRINTZ, 2011).

Un altro sintomo è l'irritazione, che appare nei bambini con la malattia, e i disturbi gastrointestinali, tra cui diarrea, vomito e dolore addominale, si verificano in circa un terzo dei pazienti, il che spiega il fatto che i bambini con DK sono più irritati degli altri (ALMEIDA, 2018; KAYIRAN; DINDAR; GURAKAN, 2010).

L'edema ascesso e retrofingeale è solitamente associato a una condizione fatale se non trattato correttamente. Gli otorinolaringoiatra possono trattarlo con drenaggio chirurgico dell'ascesso, prevenendo il blocco delle vie aeree (KIM; KWON, 2016; XIE et al., 2018).

La mucosite orale è rappresentata da eritema diffuso della mucosa, arrossamento delle labbra e della lingua e ipertrofia delle papille linguali con successivo sviluppo

RC: 82507

Disponível em: https://www.nucleodoconhecimento.com.br/salute/malattia-dikawasaki 
della lingua fragola, e di solito si verifica nella fase acuta della malattia e della convalescenza, come conseguenza del trattamento farmacologico, e impedisce al paziente di nutrirsi correttamente a causa delle lesioni, influenzando la sua adeguata alimentazione (SCARDINA et al. , 2007).

\section{LA DIAGNOSI DI DK}

DK rappresenta una sfida da diagnosticare precocemente, in quanto si tratta di un disturbo reumatologico insolito che richiede un intervento specifico al fine di evitare sequele gravi o fatali (ALMEIDA et al., 2010).

La clinica della malattia non è chiara, essendo comune la diagnosi iniziale di altre malattie, come scarlattina, estentema virale o condizioni allergiche, a causa dei sintomi iniziali della malattia. Anche con il completo adempimento dei criteri, è frequente ritardare la diagnosi corretta, che porta a un ritardo all'inizio del trattamento appropriato, favorendo la comparsa di complicanze, che possono rapidamente aggravare le condizioni cliniche del paziente. Inoltre, ci sono forme incomplete o atipiche, che rendono la diagnosi ancora più difficile. La diagnosi precoce della malattia è importante, poiché il trattamento in fase acuta riduce il processo infiammatorio nelle arterie coronarie e previene la trombosi, riducendo le possibilità di mortalità (FERRONATO et al., 2010).

Una diagnosi clinica tempestiva è essenziale per stabilire questo trattamento precoce ed evitare lesioni coronarie. Questo tipo di lesione può causare ischemia o infarto del miocardio, che è estremamente grave (KAYIRAN; DINDAR; GURAKAN, 2010; SOTELO; GONZÁLEZ, 2007).

I criteri diagnostici della malattia classica del DK sono febbre con cinque giorni o più, oltre ad almeno altri quattro criteri, che sono: eritema palmoplantare con o senza edema doloroso o sfaldamento periunigueale; Esa tema polimorfico; iperemia congiuntivale bulbare su entrambi i lati, non essudativa; Cambiamenti nelle labbra e

RC: 82507

Disponível em: https://www.nucleodoconhecimento.com.br/salute/malattia-dikawasaki 
nella mucosa orale; Linfoadenopatia cervicale - diametro superiore a $1,5 \mathrm{~cm}$, duro e indolore alla palpazione. La diagnosi di DK incompleto può essere stabilita in presenza di tre o più dei seguenti criteri: Albumina $\leq 3 \mathrm{~g} / \mathrm{dL}$; Anemia; Elevazione dell'alanina amminotransferasi; Piastrine dopo sette giorni $\geq 450.000 / \mathrm{mm} 3$; Leucociti $\geq 15.000 / \mathrm{mm}^{2}$; Urina $\geq 10$ leucociti (CASTRO; URBANO; COSTA, 2009; RODRIGUES et al., 2017; YAP; LIN; WANGUI, 2012).

Le principali sequele della malattia sono correlate al sistema cardiaco, quindi, l'imaging cardiaco mediante ecocardiografia è una parte rilevante della valutazione dei pazienti con DK sospetto. Per i casi semplici, l'ecocardiografia è raccomandata alla diagnosi, da 6 a 8 settimane dopo l'insorgenza della malattia (ATIK et al., 2017; KAYIRAN; DINDAR; GURAKAN, 2010).

Le alterazioni abortive più frequenti sono il numero di sangue con leucocitosi e neutrofilia, oltre ad aumentare $\mathrm{i}$ valori dei test di fase acuta, come la velocità di emosedimentazione e la proteina C-reattiva. La malattia viene spesso diagnosticata solo dopo il coinvolgimento dell'arteria coronarica (FERRONATO et al., 2010).

Uno studio di Kentsis et al. (2013) ha rilevato che i marcatori filamina C e meprina A sono stati rilevati sia nel siero che nelle urine dei due gruppi indipendenti di pazienti con DK partecipanti. Lo stesso studio rileva che l'uso di questi marcatori per eseguire la diagnosi di DK è più efficiente dell'uso di marcatori attualmente utilizzati (KENTSIS et al., 2013).

\section{ESAMI D'IMMAGINE E COMPLEMENTARI}

L'ecocardiogramma è uno dei principali test utilizzati per la diagnosi di DK, poiché non invasivo, ha un'elevata sensibilità e specificità per analizzare e rilevare anomalie nelle arterie coronarie, valutare il miocardio e verificare la presenza di pericardite. In questo esame, possono apparire alcuni risultati che indicano l'arterite coronarica, che precede la formazione di aneurismi, come la luminosità perivascolare, l'ectasia o il

RC: 82507

Disponível em: https://www.nucleodoconhecimento.com.br/salute/malattia-dikawasaki 
restringimento delle arterie coronarie. Nell'analisi delle arterie coronarie, il diametro interno del vaso deve essere valutato; il numero, la posizione e il tipo (sacculare o fusiforme) degli aneurismi; e la presenza o meno di trombo intraluminale e sustesi. Nella fase acuta della malattia, alcuni risultati frequenti negli ecocardiogrammi sono: riduzione della contrattilità del ventricolo sinistro, rigurgito della valvola mitrale ed effusione pericardica (CASTRO; URBAN; COSTA, 2009; MAGELLANO; ALVES, 2017; RODRIGUES et al., 2017).

Altri test che possono essere eseguiti, in base a disponibilità, gravità, vantaggi e indicazioni che variano a seconda dei casi. Ad esempio, risonanza magnetica (RNM), angioresonanza, radiografia toracica, tomografia computerizzata ad alta risoluzione, angiografia (CASTRO; URBANO; COSTA, 2009).

L'angiografia TC, un esame invasivo che presenta rischi e l'angioresonanza vengono solitamente eseguite in quei pazienti che hanno presentato anomalie su elettrocardiogramma, ecocardiografico o sintomi di ischemia miocardica (CASTRO; URBANO; COSTA, 2009; MAGALHÃES; ALVES, 2017).

Ultimamente, la tomografia computerizzata ad alta risoluzione è stata più indicata, specialmente se confrontata con la risonanza prima, poiché la sua esecuzione è più veloce e più facile da interpretare, mentre la risonanza grafia richiede un tempo anestetico più lungo di bambini per la raccolta (CASTRO; URBANO; COSTA, 2009).

La radiografia toracica, che di solito non presenta alterazioni, in circa il $15 \%$ dei casi vengono scoperte anomalie, come: modello di infiltrato reticologranare o peribronchiale, accumulo di troppo liquido nello spazio tra le pleure viscerali e parietali o collasso del polmone. Appaiono dopo il $10^{\circ}$ giorno della malattia e sono possibili controllare le radiografie da 10 a 50 giorni dopo l'inizio della condizione (CASTRO; URBANO; COSTA, 2009).

RC: 82507

Disponível em: https://www.nucleodoconhecimento.com.br/salute/malattia-dikawasaki 
Nei test di laboratorio, i risultati non sono specifici della DK, ma piuttosto un'indicazione di un alto processo infiammatorio ed è possibile trovare, all'inizio della fase acuta, un aumento della velocità di emosedimentazione (VHS), proteina Creattiva (PCR) e tassi di alfaglicoproteina acida. Nell'esame del sangue è possibile controllare una quantità anormalmente elevata di neutrofili, chiamata leucocitosi con neutrofilia, e può essere accompagnata da un aumento dei pipistrelli o eosinofilia. Inoltre, la quantità di piastrine è solitamente normale nella fase acuta, tuttavia, è possibile che in alcuni casi ci sia trombocitopenia, che è associata allo sviluppo di grave malattia coronarica e infarto del miocardio (MAGALHÃES; ALVES, 2017).

\section{ANALISI DELLE PRINCIPALI COMPLICANZE ASSOCIATE A DK}

La complicanza più grave della malattia è la vasculite coronarica, che colpisce il 15$20 \%$ dei pazienti non trattati, che può portare ad aneurismi, ectasie e sustesi delle arterie coronarie, che rappresentano il 2\% della mortalità. Altre complicazioni di DK sono state descritte come aneurismi in altre arterie come l'aorta; l'aorta addominale; arteria ascellare; arteria brachiocefalica; arterie iliache, arterie femorali e arterie renali, il che indica come DK possa influenzare enormemente il corpo umano, e quindi essere estremamente pericoloso (ALVES, et al., 2011; CHBEIR et al., 2018; MUTLUER; ÇELIKER, 2019; PRINTZ, 2011; XIE et al., 2018).

Alcuni pazienti con DK sono suscettibili alla dilatazione coronarica progressiva. Tale dilatazione coronarica si verifica durante la fase acuta e può procedere alla fase subacuta, che aggrava le condizioni cliniche di diversi pazienti (BRIEDÉ et al., 2015; 2015; LIU et al., 2017).

Possono verificarsi complicazioni gastrointestinali in DK come ostruzione intestinale, edema del colon, ischemia intestinale, pseudo-ostruzione intestinale e addome acuto, che colpisce il paziente in modo allarmante, poiché queste complicazioni possono portare alla sepsi e persino alla morte. Inoltre, le alterazioni oftalmologiche associate a DK possono includere uveite, iridociclite, emorragia congiuntivale,

RC: 82507

Disponível em: https://www.nucleodoconhecimento.com.br/salute/malattia-dikawasaki 
neurite ottica, amaurosi e ostruzione dell'arteria oculare, che causa sequele irreparabili più volte. DK si manifesta anche come vasculite necrotizzante, portando così alla perdita del tessuto interessato (ALVES, et al., 2011).

Le complicanze neurologiche dovute a lesioni al SNC possono essere le seguenti: meningoencefalite, raccolta subdurale, ipoperfusione cerebrale, ischemia, infarto cerebrale, convulsioni, emiplegia, confusione mentale, letargia e persino coma o infarto cerebrale. Tali complicazioni possono diventare irreversibili, danneggiando così per sempre la vita del paziente (ALVES, et al., 2011).

II rischio di complicanze tardive di DK suggerisce la necessità di un follow-up a lungo termine oltre all'infanzia (GIACCHI et al., 2014).

\section{TRATTAMENTI DISPONIBILI PER LE PERSONE AFFAFFATO DA DK}

II trattamento più indicato è l'applicazione di IVIG (Immunoglobulina endovenosa) in una singola dose di $2 \mathrm{~g} / \mathrm{kg}$ per infusione di 12 ore. In base all'evoluzione del quaro clinico del paziente, la dose di IVIG può essere ripetuta o possono essere aggiunti corticosteroidi, specialmente nei casi refrattari. Sono raccomandati altri farmaci, come ciclofosfamide, ciclosporina e ulinastatina. Inoltre, si raccomanda un prodotto a base di anticorpi monoclonali contro il fattore alfa della necrosi tumorale, così come un anticorpo monoclonale, noto come Abciximab, e che può trattare gli aneurismi più rapidamente (ATIK, 2007; FERRONATO et al., 1010; KAYIRAN; DINDAR; GURAKAN, 2010; PHILIP et al., 2017; SOTELO; GONZÁLEZ, 2007).

Circa il $10-15 \%$ dei pazienti con DK non mostra alcun miglioramento con il trattamento IVIG, che è il trattamento standard (MEHNDIRATTA et al., 2014). Nei pazienti pediatrici con KD, alte dosi di acido acetilsalicilico (AAS) forniscono azione antinfiammatoria, mentre basse dosi hanno un effetto inibitorio dell'aggregazione piastrinica. Pertanto, nella fase acuta della malattia, viene utilizzato ASA $80-100 \mathrm{mg} /$ $\mathrm{kg} /$ giorno, che è diviso in quattro ingestione durante il giorno, al fine di intensificare

RC: 82507

Disponível em: https://www.nucleodoconhecimento.com.br/salute/malattia-dikawasaki 
l'effetto antinfiammatorio di IVIG. Mentre in alcuni ospedali la dose di AAS viene ridotta dopo 2-3 giorni in assenza di febbre, in altri centri sanitari il trattamento persiste per un massimo di 2 settimane. Inoltre, i bambini con anomalie cardiache hanno una prescrizione per l'udito a tempo indeterminato (ATIK, 2007; CASTRO; URBANO; COSTA, 2009; MEHNDIRATTA et al., 2014).

Vengono utilizzati anche farmaci immunosoppressivi come la ciclofosfamide, insieme al prednisone o al metilprednisolone, per migliorare alcuni sintomi, come la febbre, ma questi farmaci non hanno un effetto terapeutico sulle anomalie cardiache (CASTRO; URBANO; COSTA, 2009; PHILIP et al., 2017).

Nella fase acuta della malattia DK, c'è un rischio chirurgico più elevato perché, in presenza di infiammazione, l'anastomosi con arteria coronarica infiammata corre il rischio di ostruzione. L'approccio chirurgico deve essere limitato ai pazienti con aneurismi giganti o processi ischemici dopo la fase acuta (ATIK, 2007; SOTELO; GONZÁLEZ, 2007).

La rivascolarizzazione chirurgica delle lesioni coronarie secondarie al DK è relativamente rara. Secondo le attuali linee guida, la rivascolarizzazione arteriosa completa deve essere utilizzata in giovani pazienti con piccole comorbilità al fine di ottenere una buona permeabilità all'innesto a lungo termine (BARCA et al., 2019).

Oltre alla chirurgia di rivascolarizzazione del miocardio, che è stato il trattamento preferito per le complicanze coronarie trombotiche di DK, l'intervento coronarico percutaneo (ICP) con stent regolari o percutanei con politetrafluoroetilene (PTFE), impianto di bobine, rivascolarizzazione coronarica transluminale percutanea (PTCR) con agenti trombolitici nell'arteria correlata all'infarto e ablazione coronarica percutanea (PTCRA), vengono utilizzati nei pazienti affetti dalla malattia (MUTLUER; ÇELIKER, 2019).

RC: 82507

Disponível em: https://www.nucleodoconhecimento.com.br/salute/malattia-dikawasaki 
Per quanto riguarda la gestione anestetica, ci sono poche informazioni descritte, tuttavia, la rivascolarizzazione miocardica con anestesia eseguita da gas alogenati presenta meno danni, mostrando così un migliore recupero postoperatorio della funzione miocardica rispetto agli anestetici endovenosi. Pertanto, favorisce l'inibizione della risposta neuroendocrina allo stress e prepara anche il paziente per un'estorazione precoce nel periodo postoperatorio, che aiuta nella prevenzione di possibili complicazioni derivanti dall'intubazione (MARTíNEZ; MÉNDEZ, 2013).

\section{CONCLUSIONE}

Il verificarsi di DK è variabile, essendo più presente nella regione Asia, specialmente nei bambini sotto i 5 anni di età. In America Latina l'incidenza è meno frequente e la mortalità di questa patologia è bassa.

L'agente eziologico di DK non è stato identificato, ma ci sono alcuni agenti patogeni che possono essere correlati a questa malattia. Ci sono prove che questa malattia ha una relazione genetica, questo è stato trovato a causa dell'aumento dell'incidenza tra le persone asiatiche e tra i parenti dei pazienti affetti da DK, inoltre, ci sono anche alcuni geni associati a questa patologia.

Ci sono alterazioni associate a DK come infiammazioni sistemiche, che colpiscono alcuni organi causati da angiite, meningite sterile, polmonite, adenofinite ed epatite. DK colpisce organi e arterie, principalmente vasi di medio calibro, come quelli del cuore, in modo che porti alla formazione di aneurismi, può influenzare gli occhi, le mucose della cavità orale, il polmone, l'intestino, la cistifellea e il sistema nervoso, il sistema linfatico, il sistema cutaneo e le lesioni possono verificarsi nelle labbra.

II quadro clinico del DK ha tre fasi. Lo stadio febbrile acuto è caratterizzato da congestione congiuntivale, mucosite orale, eritema, sfaldamento, eruzione polimorfica e linfoadenopatia laterocervicale. Lo stadio subacuto si verifica alla fine della febbre, i pazienti hanno la pelle che sfalda sugli arti, artrite, artralgia e

RC: 82507

Disponível em: https://www.nucleodoconhecimento.com.br/salute/malattia-dikawasaki 
trombocitosi. La fase di convalescenza si verifica quando i sintomi stanno quasi scomparendo e continuano fino alla loro normalizzazione.

Il ritardo nella corretta diagnosi di DK è abbastanza frequente, il che può portare a un ritardo nell'inizio del trattamento, che può causare complicazioni per peggiorare la condizione clinica. La diagnosi clinica precoce è estremamente importante per stabilire un trattamento precoce ed evitare lesioni coronarie.

Il trattamento di DK si basa sull'evoluzione del quadro clinico del paziente. La terapia più utilizzata è l'applicazione di IGIV, se si verificano cambiamenti in clinica, la dose di IGIV può essere ripetuta o possono essere aggiunti corticosteroidi. Possono essere indicati altri medicinali, come ciclofosfamide, ciclosporina e ulinastatina. Si raccomanda inoltre di utilizzare un prodotto a base di anticorpi monoclonali Infliximab, immunosoppressori come ciclofosfamide o ciclosporina e prednisone o metilprednisolone. II trattamento chirurgico viene eseguito principalmente nella fase acuta di DK, ma deve essere limitato a pazienti con aneurismi giganti o processi ischemici dopo la fase acuta della malattia.

\section{RIFERIMENTI}

ALMEIDA, F. C. Avaliação das manifestações clínicas e achados laboratoriais em 301 pacientes com doença de Kawasaki: acompanhamento de dez anos. Tese (Doutorado em Ciências Médicas). Faculdade de Medicina, Universidade de Brasília, Brasília. 2018.

ALMEIDA, M. A. A. L. S. Avaliação da calprotectina e dos anticorpos anticitoplasma de neutrófilos como marcadores de inflamação e autoimunidade nas diferentes fases da doença de Kawasaki. Dissertação (Mestrado em Ciências Farmacêuticas). Faculdade de Ciências da Saúde, Universidade de Brasília, Brasília. 2017.

RC: 82507

Disponível em: https://www.nucleodoconhecimento.com.br/salute/malattia-dikawasaki 
ALMEIDA, R. G. et al. Perfil da doença de Kawasaki em crianças encaminhadas para dois serviços de reumatologia pediátrica do Rio de Janeiro, Brasil. Revista Brasileira de Reumatologia, v. 50, n. 5, p. 529-538, set./out. 2010.

ALVES, N. R. M. et al. Estudo prospectivo das complicações da Doença de Kawasaki: análise de 115 casos. Revista da Associação Médica Brasileira, v. 57, n. 3, p. 299-305, mar. 2011.

ATIK, E. Doença de Kawasaki: Regressão de Aneurismas Gigantes das Artérias Coronárias com Obstrução Tardia Posterior Kawasaki Disease: Giant Coronary Arteries Aneurysms Regression and Later Stenosis. Arquivos brasileiros de Cardiologia, v. 88, n. 1, p. 22-23, 2007.

ATIK, E.; FORONDA, A.; BUSTAMANTE, L. N. P. Kawasaki Disease. Involution of Giant Coronary Aneurysms After Prolonged Anti-inflammatory Treatment. Arquivos brasileiros de Cardiologia, v. 81, n. 3, p. 265-72, set. 2003.

ATIK, E. et al. Caso 6/2017 - Extenso Aneurisma Gigante de Artéria Coronária Esquerda por Vasculite de Kawasaki em Homem Assintomático com 48 Anos de Idade. Arquivos brasileiros de Cardiologia, v. 109, n. 5, p. 489-490, nov. 2017.

BARCA, L. V. et al. Ligature of the Left Main Coronary Artery after Surgery in Kawasaki Disease: Case Report. Braz J Cardiovasc Surg, v. 34, n. 1, p. 111-113, jan./fev., 2019.

BRIEDÉ, S. et al. Hamoen M, Oosterveld M JS, Breur JMPJ. Langetermijneffecten van de ziekte van Kawasaki. Ned tijdschr geneeskd, v. 154, n. 2121, p. 1-6, out. 2015.

CASTRO, P. A.; URBANO, L. M. F.; COSTA, I. M. C. Doença de Kawasaki. Anais Brasileiros de Dermatologia, v. 84, n. 4, p. 317-331, mai. 2009.

RC: 82507

Disponível em: https://www.nucleodoconhecimento.com.br/salute/malattia-dikawasaki 
CHBEIR, D. et al. Kawasaki disease: abnormal initial echocardiogram is associated with resistance to IV $\mathrm{Ig}$ and development of coronary artery lesions. Pediatric Rheumatology Onlinw Journal, v. 6, n. 48, p. 1-10, jul. 2018.

FERRONATO, A. E. et al. Doença de Kawasaki: experiência clínica em hospital universitário. Revista Paulista de Pediatria, v. 28, n. 2, p. 148-154, jun. 2010.

GIACCHI, V. et al. Avaliação do espessamento íntimo da artéria coronária em pacientes com diagnóstico prévio de doença de Kawasaki por meio de ecocardiografia transtorácica de alta resolução: nossa experiência. BMC Cardiovasc Disord, v. 14, n. 106, p 1-6, ago. 2014.

HUANG, X. et al. Influenza infection and Kawasaki disease. Revista da Sociedade Brasileira de Medicina Tropical, v. 48, n. 3, p. 243-248, jun. 2015.

KAYIRAN, S. M.; DINDAR, A. N.; GURAKAN, B. An evaluation of children with Kawasaki disease in Istanbul: a retrospective follow-up study. Clinical Science, v. 65, n. 12, p. 1261-1265, dez. 2010.

KENTSIS, A. et al. Urine proteomics for discovery of improved diagnostic markers of Kawasaki disease. EMBO Mol Med, v. 5, n. 2, p. 210-220, fev. 2013.

KIM, J. S.; KWON, S. H. Atypical Kawasaki disease presenting a retropharyngeal abscess. Braz J Otorhinolaryngol, v. 82, n. 4, p. 484-486, 2016.

LIU, M. Y. et al. Risk factors and implications of progressive coronary dilatation in children with Kawasaki disease. BMC Pediatrics, v. 17, n. 139, p. 1-7, jun. 2017.

MAGALHÃES, C. M. E. Estudo da prevalência da perda auditiva neurossensorial como complicação da doença de Kawasaki. Tese (Doutorado em Ciências da Saúde) - Faculdade de ciências da saúde, Universidade Federal de Brasília, Brasília. 2008.

RC: 82507

Disponível em: https://www.nucleodoconhecimento.com.br/salute/malattia-dikawasaki 
MAGALHÃES, C. M. R.; ALVES, N. R. M. Doença de Kawasaki. In: I. Burns, DAR, Campos Júnior D, Silva LR, Borges WG. Tratado de pediatria: Sociedade Brasileira de Pediatria. 4. ed. Barueri, SP: Manole, 2017. p.1825-1835.

MARTÍNEZ, U. M.; MÉNDEZ, F. M. Manejo anestésico del paciente com enfermedad de Kawasaki durante la cirugía de revascularización coronaria: informe de um caso. Archivos de Cardiología de México, v. 83, n. 4, p. 267-272, abr. 2013.

MEHNDIRATTA, S. et al. A case of incomplete and refractory Kawasaki disease: Diagnostic and therapeutic challenges. The South African Journal of Child Health, v. 8, n. 1, p. 37-38, fev. 2014.

MUTLUER, F. O.; ÇELIKER, A. Comment on Ligature of the Left Main Coeonary Artery after Surgery in Kawasaki Disease: Case Report. Brazilian Journal of Cardiovascular Surgery, v. 34, n.3, p. 382, jul. 2019.

PHILIP, S. et al. Role of Antioxidants in Horse Serummediated Vasculitis in Swine: Potential Relevance to Early Treatment in Mitigation of Coronary Arteritis in Kawasaki Disease. Pediatrics and Neonatology, v. 58, n. 4, p. 328-337, fev. 2017.

PRINTZ, B. F. Noncoronary Cardiac Abnormalities Are Associated With Coronary Artery Dilation and With Laboratory Inflammatory Markers in Acute Kawasaki Disease. Journal of the American College of Cardiology, v. 57, n. 1, p. 68-92, jan. 2011.

RODRIGUES, M. et al. Doença de Kawasaki e Complicações Cardiovasculares em Pediatria. Birth and growth medical jornal, v.27, n. 1, p. 54-58, fev. 2017.

ROSSI, F. S. et al. Extensa linfadenite cervical mimetizando adenite bacteriana como primeira manifestação da doença de Kawasaki. Einstein, v. 13, n. 3, p. 426429, jul./set. 2015.

RC: 82507

Disponível em: https://www.nucleodoconhecimento.com.br/salute/malattia-dikawasaki 
ROWLEY, A. H. et al. Allograft Inflammatory Factor-1 Links T-Cell Activation, Interferon Response, and Macrophage Activation in Chronic Kawasaki Disease Arteritis. Journal of the Pediatric Infectious Diseases Society, v. 6, n. 3, p. 94102, set. 2017.

SCARDINA, G. A. et al. Oral necrotizing microvasculitis in a patient affected by Kawasaki disease. Medicina Oral Patologia Oral y Cirugia Bucal, v. 12, n. 8, p. 560-564, dez. 2007.

SOTELO, N.; GONZÁLEZ, L. A. Kawasaki disease: A rare pediatric pathology in Mexico Twenty cases report from the Hospital Infantil del Estado de Sonora. Archivos de Cardiologia de Mexico, v. 77, n. 4, p. 299-307, dez. 2007.

XIE, X. F. et al. Proteomics study of serum exosomes in Kawasaki disease patients with coronary artery aneurysms. Cardiology Journal, v. 26, n. 5, p. 584-593, fev. 2018.

YAP, C. Y.; LIN, L. H.; WANGI, N. K. An atypical presentation of Kawasaki disease: a 10- year-old boy with acute exudative tonsillitis and bilateral cervical lymphadenitis. CLINICS, v. 67, n. 6, p. 689-692, 2012.

Inviato: Aprile 2021.

Approvato: Aprile 2021.

RC: 82507

Disponível em: https://www.nucleodoconhecimento.com.br/salute/malattia-dikawasaki 